\title{
Array-based comparative genomic hybridization of mapped BAC DNA clones to screen for chromosome 14 copy number abnormalities in meningiomas
}

\author{
Ana Belén Espinosa ${ }^{1}$, Carlos Mackintosh ${ }^{2}$, Angel Maíllo ${ }^{3}$, Laura Gutierrez ${ }^{4}$, \\ Pablo Sousa ${ }^{3}$, Marta Merino ${ }^{3}$, Javier Ortiz ${ }^{5}$, Enrique de Alava $^{2}$, Alberto Orfao ${ }^{4}$ \\ and María Dolores Tabernero ${ }^{\star, 1,6}$
}

\author{
${ }^{1}$ Unidad de Investigación, Hospital Universitario de Salamanca, Salamanca, Spain; ${ }^{2}$ Laboratory of Molecular \\ Pathology, Centro de Investigación del Cáncer-IBMCC, Universidad de Salamanca-CSIC, Salamanca, Spain; \\ ${ }^{3}$ Neurosurgery Service, Hospital Universitario de Salamanca, Salamanca, Spain; ${ }^{4}$ Centro de Investigación del \\ Cáncer-IBMCC (CSIC-USAL), Cytometry General Service and Department of Medicine, University of Salamanca, \\ Salamanca, Spain; ${ }^{5}$ Pathology Service, Hospital Universitario de Salamanca, Salamanca, Spain; ${ }^{6}$ IECSCYL-Hospital \\ Universitario de Salamanca, Salamanca, Spain
}

Chromosome 14 loss in meningiomas are associated with more aggressive tumour behaviour. To date, no studies have been reported in which the entire chromosome $14 \mathrm{q}$ of meningioma tumour cells has been studied by high-resolution array comparative genomic hybridization (a-CGH). Here, we used a high-resolution a-CGH to define the exact localization and extent of numerical changes of chromosome 14 in meningioma patients. An array containing 807 bacterial artificial chromosome clones specific for chromosome 14q (average resolution of $\sim 130 \mathrm{~Kb}$ ) was constructed and applied to the study of 25 meningiomas in parallel to the confirmatory interphase fluorescence in situ hybridization (iFISH) analyses. Overall, abnormalities of chromosome $14 q$ were detected in $10 / 25$ cases $(40 \%)$. Interestingly, in seven of these cases, loss of chromosome 14q32.3 was detected by iFISH and confirmed to correspond to monosomy 14 by a-CGH. In contrast, discrepant results were found between iFISH and a-CGH in the other three altered cases. In one patient, a diploid background was observed by iFISH, while monosomy 14 was identified by a-CGH. In the remaining two cases, which showed gains of the IGH gene by iFISH, a-CGH did not detected copy number changes in one case showing a tetraploid karyotype, while in the other tumour, varying genetic imbalances along the long arm of chromosome 14 were detected. In summary, here, we report for the first time, the high-resolution a-CGH profiles of chromosome $14 q$ in meningiomas, confirming that monosomy 14 is the most frequent alteration associated with this chromosome; other numerical abnormalities being only sporadically detected.

European Journal of Human Genetics (2008) 16, 1450-1458; doi:10.1038/ejhg.2008.128; published online 16 July 2008

Keywords: chromosome 14; array $\mathrm{CGH}$; BAC probes; loss; gain; meningioma

\footnotetext{
*Correspondence: Dr MD Tabernero, Unidad de Investigación, Hospital Universitario de Salamanca, Paseo de San Vicente 58, Salamanca 37007, Spain. Tel: + 349232912 30; Fax: + 349232946 24;

E-mail: taberner@usal.es

Received 4 March 2008; revised 16 May 2008; accepted 19 June 2008; published online 16 July 2008
}

Introduction

Among other chromosomal abnormalities, meningioma patients frequently display alterations of chromosome $14 \mathrm{q}$ as detected by both cytogenetic and molecular techniques. ${ }^{1-5}$ In recent years, evidence has accumulated about the existence of a clear association between chromosome 
14 loss and male sex, ${ }^{6}$ as well as a more aggressive behaviour of the disease and a shorter recurrence-free survival. ${ }^{1-3,5,7-10}$ Overall, these findings suggest that one or more tumour suppressor genes might be responsible for the adverse prognostic impact of chromosome 14 loss in meningioma patients, the specific relevant genes remaining to be identified. This could be, at least in part, because of the fact that no studies have characterized in detail the chromosome 14 imbalances occurring in meningiomas, through the use of high-resolution genetic/molecular approaches. Despite this, the inactivation of NDRG2 gene, localized at the chromosome $14 \mathrm{q} 11.2$ region, has been recently reported in histologically malignant meningiomas. $^{11}$

Array-based comparative genomic hybridization (a-CGH) is a powerful technique developed to detect copy number variation $(\mathrm{CNV})$ of specific DNA sequences with higher resolution $^{12}$ than the conventional approaches - for example, conventional karyotyping and metaphase CGH-. ${ }^{2,13-17}$ This assay is based on a competitive hybridization between tumour and control DNA, labelled with different fluorochromes, to previously defined chromosome sequences inserted into well-characterized genomic clones - for example, bacterial (BAC) or P1-derived artificial chromosomes (PAC) - spotted onto glass slides ${ }^{18-20}$ with a resolution of around between $100 \mathrm{~Kb}$ and $1 \mathrm{Mb}^{21-23}$

In this study, we have built an a-CGH to get detailed information about $\mathrm{CNV}$ involving specific DNA sequences of chromosome $14 \mathrm{q}$ in a series of 25 meningioma tumours. Our major goal was to validate previous results about chromosome $14 \mathrm{q}$ abnormalities detected in meningiomas, usually through the use of an interphase fluorescence in situ hybridization (iFISH) probe directed to the IGH gene locus at $14 \mathrm{q} 32.3,{ }^{5}$ and to finely map DNA imbalances of chromosome $14 \mathrm{q}$ from these patients, by using 807 different DNA clones aimed at mapping the $107 \mathrm{Mb}$ of chromosome 14. To our knowledge, this is the first study in which chromosome 14 abnormalities have been analysed in meningiomas using high-resolution a-CGH. Our results confirm that the entire loss of chromosome 14 is the most frequent alteration in these tumours, although distinct and varying changes may be observed in specific cases.

\section{Materials and methods Patients and samples}

A total of 25 meningioma samples from 24 different individuals who underwent diagnostic curative surgery at the Neurosurgery Service of the University Hospital of Salamanca, between February 2003 and November 2006, were randomly selected from a larger series of 171 patients to be included in this study. Twelve of the 25 samples used in this study had been also included in a previous report. ${ }^{10}$ All tumour samples but one corresponded to primary tumours; the other sample was from a recurrent menin- gioma. In all patients, diagnosis and classification of meningioma were performed according to the WHO criteria. ${ }^{24}$ The most relevant clinical and histopathological characteristics of the cases analysed are shown in Table 1.

In addition, the following samples were used as reference DNA: (a) a DNA pool from peripheral blood (PB) leucocytes from healthy female $(n=25)$ and male $(n=15)$ volunteers from National DNA Bank of Spain, Salamanca, Spain (www.bancoadn.org); (b) constitutional DNA from each of the meningioma patients studied and (c) DNA from normal meningeal female $(n=4)$ cells, kindly provided by C Caballero and T Tuñón from the Neurological Tissue Bank of Navarra (Pamplona, Spain). In all cases, both tumour DNA and normal reference DNA were extracted using the QIAmp DNA Mini Kit (Qiagen, Hilden, Germany), according to the instructions of the manufacturer.

The study was approved by the ethical committee of the local University Hospitals of Salamanca and Navarra and samples were obtained only after the informed consent was given by the individual.

\section{Array comparative genomic hybridization studies}

Full genomic coverage clones for chromosome $14 \mathrm{q}$ provided by the BACPAC Resources Children's Hospital Oakland Research Institute (http://bacpac.chori.org) were used for a-CGH analyses. These included a total of 807 DNA clones with a median size of $200 \mathrm{~Kb}$ and an average spacing of $\sim 130 \mathrm{~Kb}$, as defined by the overlapping BAC segments. In addition, $110 \mathrm{BAC}$ and PAC clones mapping to different regions of human chromosome $\mathrm{X}$ (Welcome Trust; Sanger Institute, Cambridge, UK) and 16 different autosomal chromosomes other than chromosome 14 were also used in parallel to chromosome $14 \mathrm{BAC}$ clones for data normalization. Clone position along the chromosomes was determined using the UCSC genome browser (http:// genome.ucsc.edu).

After growing the clones in 2XYT culture medium, automated extraction of the clones' DNA was performed using the R.E.A.L. Prep BioRobot kit (Qiagen) and the BioRobot 3000 (Qiagen). Afterward, DNA from each BAC clone was amplified by DOP-PCR as described previously. ${ }^{20}$ The amplified DNA sequences were then spotted (spot diameter of approximately $150 \mu \mathrm{m}$ ) in triplicate onto CodeLink Activated Slides (GE Healthcare, Fairfield, CT), using the MicroGrid II arrayer (BioRobotics, Cambridge, UK). Before DNA hybridization, $500 \mathrm{ng}$ of both tumour genomic DNA and reference DNA were labelled by random priming with Cy5 dCTP and Cy3 dCTP, respectively (GE Healthcare), precipitated with $100 \mu$ g of Cot-1 DNA (Roche Diagnostics, Mannheim, Germany) and dissolved in hybridization buffer. Hybridization of the array - blocked with $80 \mu \mathrm{g}$ of herring sperm DNA (Sigma) and $100 \mu \mathrm{g}$ of Cot-1 DNA - was performed in a moist chamber at $42^{\circ} \mathrm{C}$ for $48 \mathrm{~h}$. Afterward, the array was scanned in the GenePix 4000B scanner (Axon Instruments Inc., Union City, CA). 
Table 1 Chromosome 14 status in meningioma tumours $(n=25)$ as analysed by both iFISH and array CGH and its relationship with other clinical and histopathological disease characteristics

\begin{tabular}{|c|c|c|c|c|c|c|c|c|c|c|}
\hline $\begin{array}{l}\text { Case } \\
I D\end{array}$ & Gender & $\begin{array}{l}\text { Age at diagnosis } \\
\text { or recurrence } \\
\text { (years) }\end{array}$ & $\begin{array}{l}\text { Tumour } \\
\text { localization }\end{array}$ & $\begin{array}{l}\text { Tumour } \\
\text { histopathology }\end{array}$ & Recurrence & $\begin{array}{c}\text { RFS } \\
\text { (months) }\end{array}$ & $\begin{array}{l}\text { Monosomy } 14 q \\
\text { in the ancestral } \\
\text { tumour cell clone }\end{array}$ & $\begin{array}{l}\text { Chromosor } \\
\text { iFISH } \\
(14 q 32.3 \\
\text { probe) }\end{array}$ & Array $\mathrm{CGH}$ & $\begin{array}{l}\text { iFISH } \\
14 q \text { BAC } \\
\text { clone probes }^{\mathrm{a}}\end{array}$ \\
\hline 3 & Female & 76 & Tentorial & Transitional & NR & 54 & No & Diploid & Diploid & Diploid \\
\hline 4 & Female & 68 & Convexity & Psammomatous & NR & 55 & $\mathrm{No}^{\mathrm{b}}$ & Diploid & Diploid & Diploid \\
\hline 8 & Male & 56 & Cranial base & Transitional & NR & 52 & No & Diploid & Diploid & Diploid \\
\hline 11 & Female & 68 & Cranial base & Meningotheliomatous & NR & 50 & No & Diploid & Diploid & Diploid \\
\hline 12 & Female & 36 & Spinal & Transitional & NR & 43 & No & Diploid & Diploid & Diploid \\
\hline 13 & Male & 77 & Cranial base & Meningotheliomatous & NR & 35 & No & Diploid & Diploid & Diploid \\
\hline 14 & Male & 54 & Cranial base & Fibroblastic & NR & 34 & No & Diploid & Diploid & Diploid \\
\hline 15 & Female & 54 & Cranial base & Transitional & NR & 27 & No & Diploid & Diploid & Diploid \\
\hline 16 & Female & 26 & Cranial base & Meningotheliomatous & NR & 27 & $\mathrm{No}^{\mathrm{b}}$ & Diploid & Diploid & Diploid \\
\hline 18 & Female & 65 & Spinal & Psammomatous & NR & 27 & No & Diploid & Diploid & Diploid \\
\hline 19 & Female & 53 & Tentorial & Fibroblastic & NR & 27 & No & Diploid & Diploid & Diploid \\
\hline 20 & Female & 69 & Cranial base & Meningotheliomatous & NR & 26 & No & Diploid & Diploid & Diploid \\
\hline 21 & Female & 42 & Cranial base & Meningotheliomatous & NR & 25 & No & Diploid & Diploid & Diploid \\
\hline 22 & Female & 65 & Spinal & Psammomatous & NR & 18 & No & Diploid & Diploid & Diploid \\
\hline 23 & Female & 77 & Tentorial & Meningotheliomatous & NR & 17 & No & Diploid & Diploid & Diploid \\
\hline 24 & Male & 68 & Tentorial & Meningotheliomatous & NR & 14 & Yes $^{\mathrm{b}}$ & Diploid & Monosomy & Diploid \\
\hline 1 & Male & 41 & Convexity & Meningotheliomatous & Yes & $242^{c}$ & Yes $^{\mathrm{b}}$ & Loss & Monosomy & Losses \\
\hline 5 & Female & 60 & Convexity & Meningotheliomatous & Yes & $30^{c}$ & $Y_{e s}^{b}$ & Loss & Monosomy & Losses \\
\hline $5 R^{d}$ & Female & 62 & Convexity & Meningotheliomatous & NR & 22 & Yes & Loss & Monosomy & Losses \\
\hline 6 & Female & 52 & Tentorial & Atypical & NR & 52 & Yes $^{\mathrm{b}}$ & Loss & Monosomy & Losses \\
\hline 7 & Female & 70 & Cranial base & Meningotheliomatous & NR & 52 & Yes & Loss & Monosomy & Losses \\
\hline 10 & Female & 60 & Tentorial & Transitional & NR & 50 & Yes $^{\mathrm{b}}$ & Loss & Monosomy & Losses \\
\hline 17 & Female & 84 & Tentorial & Meningotheliomatous & NR & 27 & Yes $^{\mathrm{b}}$ & Loss & Monosomy & Losses \\
\hline 2 & Female & 62 & Convexity & Meningotheliomatous & NR & 56 & $\mathrm{No}^{\mathrm{b}}$ & Gain ${ }^{e}$ & Diploid & Gain \\
\hline 9 & Male & 76 & Convexity & Meningotheliomatous & NR & 51 & $\mathrm{No}^{\mathrm{b}}$ & Gain $^{f}$ & Loss/Diploid/ & Loss/ \\
\hline & & & & & & & & & Gain & $\begin{array}{l}\text { Diploid/ } \\
\text { Gaing }\end{array}$ \\
\hline
\end{tabular}

These copy number changes were detected with the following BAC clone probes: CTD-2120P12, RP11-433O19, RP11-73E17, RP11-700L24, RP11-746C4, RP11-31E3, RP11-536A5, RP11-410E23, RP11-532E10, RP11-493E18 and RP11-84H9.

${ }^{\mathrm{b}}$ Cases with associated del(1p36) in the ancestral tumour cell clone.

${ }^{\mathrm{C}}$ Tumour recurrence occurred at this time. $\mathrm{NR}=$ no recurrence till the moment of closing this study.

${ }^{\mathrm{d}}$ Case $5 \mathrm{R}$ corresponds to the first tumour recurrence of case 5 .

Four fluorescence signals detected.

${ }^{\mathrm{f}}$ Three fluorescence signals detected.

9Losses detected with BAC probes CTD-2120P12, RP11-433019, RP11-73E17, RP11-746C4, RP11-31E3, RP11-536A5 and RP11-410E23; diploid regions shown with the RP11-700L24

BAC probe, and gain detected with the RP11-493E18 BAC probe. 

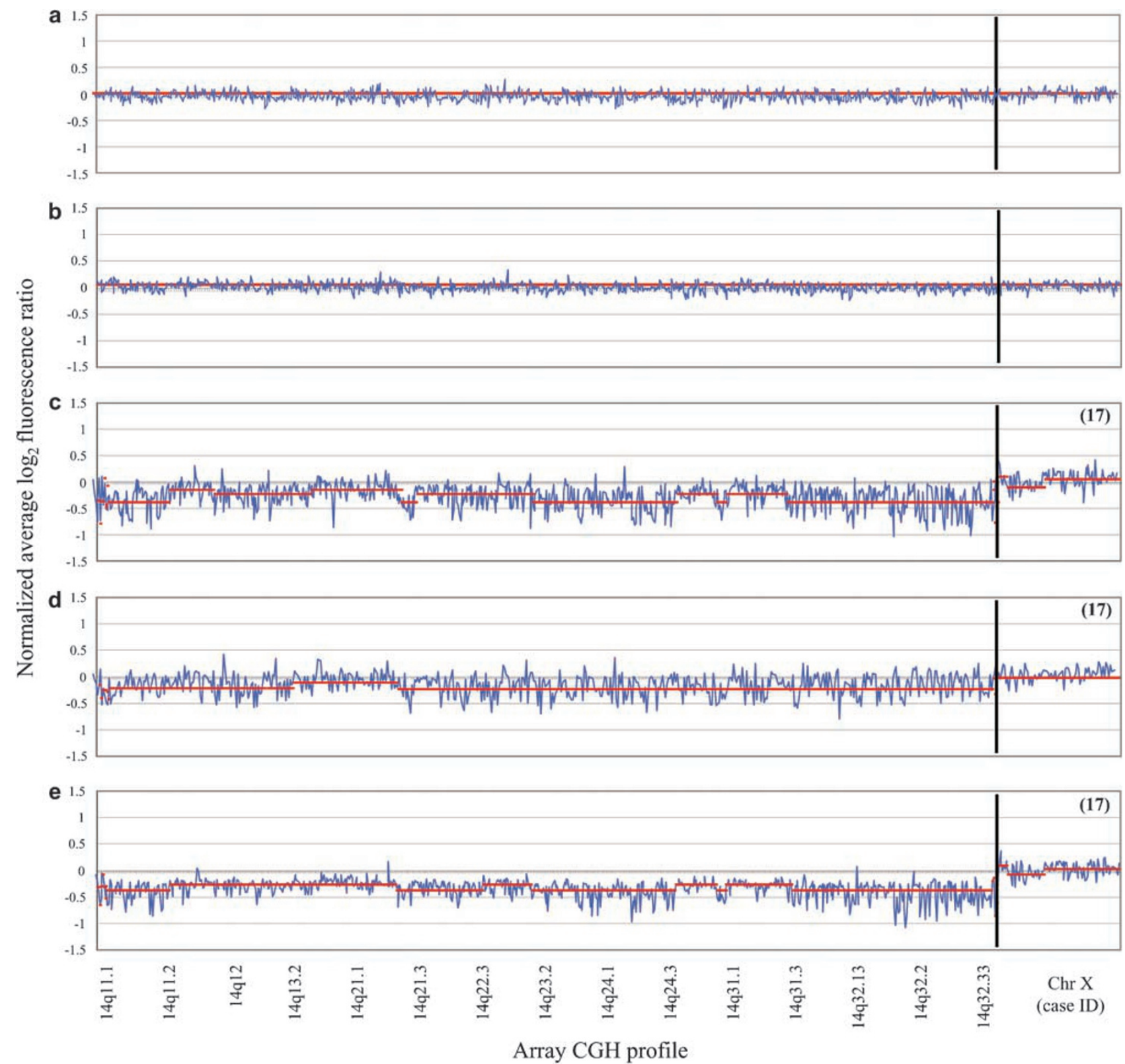

Figure 1 Illustrating high-resolution array comparative genomic hybridization (a-CGH) profiles for chromosome 14 of a female meningioma tumour representative of 25 cases studied. A-CGH profiles obtained after hybridizing DNA from a normal female vs another normal female and DNA from a normal male $v s$ another normal male are shown in (a and $\mathbf{b})$, respectively. In (c-e), female tumour DNA (case 17) was hybridized against three different reference DNA samples used to validate the hybridization method: a pool of peripheral blood DNA from 25 healthy females (c), constitutional DNA from the same meningioma patient (d) and normal female meningeal cells' DNA (e), respectively. In all plots, BAC clones used are ordered from the centromere to the telomere of chromosome 14 in the X-axis, at the left of the vertical bar; at the right of the vertical bar, the hybridization profiles obtained for control BAC clones against different regions of chromosome $\mathrm{X}$, are also shown. In the $\mathrm{Y}$-axis, the average normalized log2 ratios of the fluorescence signals detected for the three replicated spots measured in individual experiments for each BAC clone on the array are displayed. Fluctuating ratios observed in ( $c$ and $\mathbf{d}$ ) could be due to the copy number variations in chromosome 14 regions identified by specific BAC clones and/ or technical artefacts related to variability in the hybridization.

High-resolution a-CGH of each tumour sample DNA was performed against a DNA pool from healthy females (Figure 1c), constitutional DNA from the same meningioma patient (Figure 1d) and a DNA pool from normal meningeal female cells (Figure 1e). Scanned images were analysed using the GenePix Pro 4.0 software (Axon Instruments Inc.). Only those spots with clear hybridization signals and the expected size and shape were used to normalize data. Chromosome $\mathrm{X}$ clones and autosomal chromosomes other than chromosome 14 clones were used 
for data normalization. Normalized data were subsequently processed with the snapCGH bioconductor software package (CRAN - Comprehensive R Archive Network, http://cran.r-project.org-) after removing spots with an $\mathrm{SD}>0.2$ as well as those 'low-quality' spots flagged by the GenePix software. DNA copy number algorithms and Merge Levels scripts (snapCGH software package), including a $\log 2$ transformation of the $\mathrm{Cy} 5 / \mathrm{Cy} 3$ ratio values, were then applied to data corresponding to each DNA clone. A conventional $\log 2$ ratio threshold of \pm 0.25 was used to identify gains and losses of specific regions of chromosome 14. The quality of a-CGH results was tested by performing control hybridizations with the reference DNA samples described above. All control hybridizations (Figure 1a and b) were performed in parallel to the analysis of tumour samples, using the same batch of arrays under the identical labelling and hybridization conditions.

\section{iFISH studies with the 14q32 probe}

iFISH studies with the 14q32.3 probe (LSI IGH/BCL2 Dual Color, Dual Fusion Translocation Probe, Vysis Inc., Downers Grove, IL) were performed in all cases on fresh tumour samples as described elsewhere. ${ }^{5}$

\section{iFISH analysis of chromosome 14 with fluorescently labelled BAC probes}

To confirm the presence of $\mathrm{CNV}$ of specific regions of chromosome $14 \mathrm{q}$ detected by a-CGH, 11 BAC clone probes used in the array (Table 2) were fluorescently labelled by nick translation (Nick Translation Kit; Vysis Inc.) for further iFISH analyses. To assess the correct mapping of the BAC clones on chromosome 14, before hybridization with tumour cells, labelled chromosome 14 BAC probes were hybridized to normal metaphases (Vysis Inc.) using previously reported techniques. ${ }^{5}$ Those probes, which showed cohybridization in chromosomes other than chromosome 14 were discarded from further analyses. Hybridized slides were examined visually using an Olympus microscope equipped with the appropriate set of filters

Table 2 BAC clones $(n=11)$ used to validate the results of array comparative genomic hybridization $(\mathrm{a}-\mathrm{CGH})$

\begin{tabular}{lcccc}
\hline BAC clone & $\begin{array}{c}\text { Starting } \\
b p\end{array}$ & $\begin{array}{c}\text { Ending } \\
b p\end{array}$ & $\begin{array}{c}\text { Number } \\
\text { of } b p\end{array}$ & Localization \\
\hline CTD-2120P12 & 20238025 & 20398600 & 160575 & $14 \mathrm{q} 11.2$ \\
RP11-433O19 & 33950156 & 34130673 & 180517 & $14 \mathrm{q} 13.1$ \\
RP11-73E17 & 34343505 & 34485026 & 141521 & $14 \mathrm{q} 13.2$ \\
RP11-700L24 & 64190857 & 64415593 & 224736 & $14 \mathrm{q} 23.3$ \\
RP11-746C4 & 72480956 & 72642631 & 161675 & $14 \mathrm{q} 24.2$ \\
RP11-31E3 & 73034707 & 73200904 & 166197 & $14 \mathrm{q} 24.3$ \\
RP11-536A5 & 74082799 & 74277120 & 194321 & $14 \mathrm{q} 24.3$ \\
RP11-410E23 & 83142999 & 83279501 & 136502 & $14 \mathrm{q} 31.2$ \\
RP11-532E10 & 103230563 & 103442515 & 211952 & $14 \mathrm{q} 32.33$ \\
RP11-493E18 & 103877829 & 104082121 & 204292 & $14 \mathrm{q} 32.33$ \\
RP11-84H9 & 105325858 & 105501944 & 176086 & $14 \mathrm{q} 32.33$ \\
\hline
\end{tabular}

for DAPI and Spectrum Red. For each probe, only those spots $(n \geq 200)$ with similar intensity and shape were counted in areas with $<1 \%$ unhybridized nuclei.

\section{Results}

Overall, most a-CGH results were in agreement with those revealed by iFISH studies performed with the 14q32 probe (Table 1). Accordingly, a-CGH confirmed the existence of loss of chromosome $14 \mathrm{q}$ compatible with monosomy 14 (Table 1 and Figure 2a) in all seven cases showing del(14q32) by iFISH; additionally, one case displaying a diploid background by iFISH with the 14q32.3 probe, showed monosomy by a-CGH (Table 1 ). In turn, no loss of chromosome $14 \mathrm{q}$ was observed in the remaining meningioma tumours where both iFISH with the $14 \mathrm{q} 32.3$ probe and a-CGH results were compatible with a diploid $(n=15)$ content (Table 1 and Figure $2 b$ ). In contrast, among those two cases showing gains of chromosome 14 with the $14 \mathrm{q} 32$ iFISH probe, a-CGH did not detect $\mathrm{CNV}$ in one case showing a tetraploid karyotype in $35 \%$ of the nuclei, while in the other tumour, carrying $20 \%$ of cells showing trisomy 14 with the $14 \mathrm{q} 32$ iFISH probe, varying genetic imbalances along the long arm of chromosome 14 were observed (Table 1 and Figure 3a). In this later tumour, the ratio goes down between the regions covered by the 14q11.2$14 q 21.2$ and the 14q24.1-14q32.12 BAC clones. As individual clone ratios are difficult to judge, iFISH with clones RP11-678F17 to RP11-714L5 and RP11-368B1 to RP11-693P5 were applied to confirm the occurrence of a hemizygous deletion in both regions of approximately 27 and $26 \mathrm{Mb}$, respectively. Interestingly, these $14 \mathrm{q}$ deletions coexisted in this tumour with an increased number of copies of a region of approximately $11 \mathrm{Mb}$ localized distal to the deleted regions, between $14 \mathrm{q} 32.2$ and the telomere of chromosome 14q (clones RP11-306M6 to CTD-2313E3). Finally, in this same tumour, in between the two deleted $14 \mathrm{q}$ regions a diploid background was observed at the 14q21.3-q23.3 and 14q32.13 regions with a length of 21 and $3 \mathrm{Mb}$, respectively (clones RP11-6F8 to RP11-313C5 and RP11-371E8 to RP11-241N4; Table 1 and Figure 3a).

Complementary iFISH analyses were performed with a variety of chromosome $14 \mathrm{q}$ probes derived from the set of BAC clones used for the a-CGH studies (Table 2). Overall, a high degree of agreement was found between the log2 ratios obtained by a-CGH and the results of iFISH analyses performed with these probes (Table 1). Accordingly, iFISH studies showed the entire loss of chromosome $14 \mathrm{q}$ with all the BAC probes in seven of eight cases displaying monosomy 14 by a-CGH; interestingly, in the remaining case, a diploid iFISH pattern was observed for the BAC clones in line with the results obtained also with the $14 q 32.3$ iFISH probe (Table 1), with more than $90 \%$ of the tumour cells displaying two iFISH signals. In addition, a 


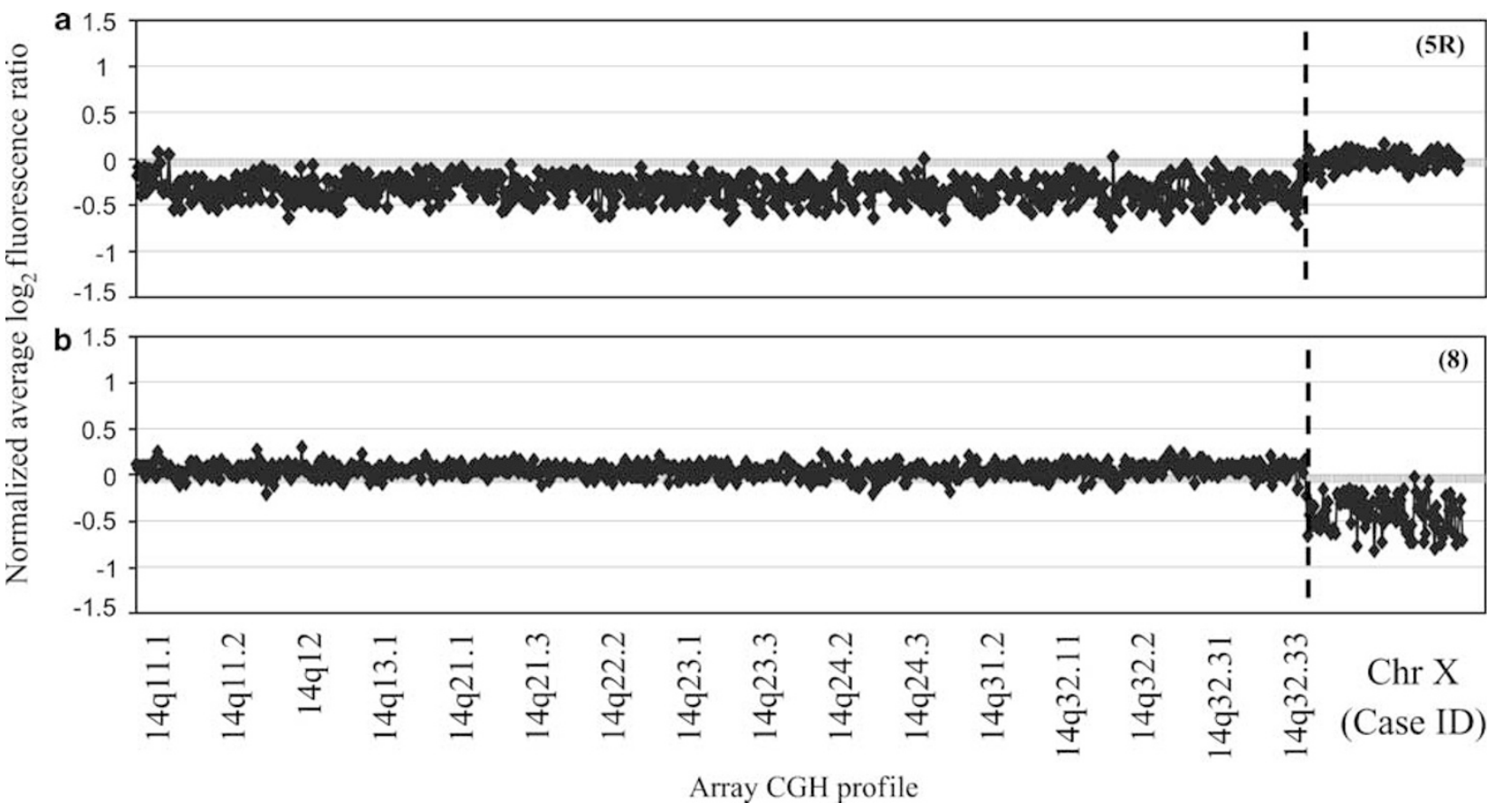

Figure 2 Illustrating a-CGH profiles of a case (case 5R) showing an abnormally reduced number of copies for all $14 \mathrm{q}$ BAC clones analysed, indicating the existence of complete loss of one copy of chromosome $14 \mathrm{q}$ (a) and a diploid meningioma tumour from a male patient (case 8 ) against reference female DNA (b). The X-axis shows chromosome 14 clones ordered from the centromere (left hand side) to the telomere (right hand side). Dots at the right of the vertical bar indicate the control clones from chromosome $\mathrm{X}$.

cell clone showing tetrasomy 14 in $35 \%$ of the nuclei was confirmed along the long arm of chromosome 14 with all the BAC probes used in a case displaying four fluorescence signals with the commercial $14 \mathrm{q} 32$ IGH gene probe and a diploid a-CGH profile. In the remaining case shown to be altered by a-CGH, the different BAC clones tested confirmed the coexistence of allelic deletions - probes: CTD-2120P12 (14q11.2), RP11-433O19 (14q13.1), RP1173E17 (14q13.2), RP11-746C4 (14q24.2), RP11-536A5 (14q24.3) and RP11-410E18 (14q31.2) with an average $\log 2$ ratio by a-CGH of $-0.42,-0.24,-0.37,-0.32,-0.26$ and -0.26 , respectively - and gains - probe RP11-493E18 (14q32.33) with an average $\log 2$ ratio by a-CGH of $0.42-$ with normal number of copies for the RP11-700L24 (14q23.3) BAC clone - average $\log 2$ ratio of 0.06- (Table 1 and Figure 3). In turn, all other 15 cases showing normal diploid a-CGH profiles showed also a diploid content for the iFISH probes used.

\section{Discussion}

Loss of chromosome 14 has been recurrently reported in a significant proportion of meningiomas. From the clinical point of view, chromosome 14 loss has been associated with a shorter recurrence-free survival, independently of tumour histopathology, particularly when detected in all tumour cells in the sample (eg, in the ancestral tumour cell clone) in association with $\operatorname{del}(1 \mathrm{p} 36) .^{3,9,10,25}$ Altogether, these results suggest the presence of one or more tumour suppressor genes in this chromosome. Despite this, few studies have been reported so far in which a more precise characterization of the genetic changes involving chromosome 14, aimed at a better understanding of the clinical behaviour of the tumour, have been analysed. Highresolution a-CGH has been applied to the study of meningiomas focusing on the analysis of chromosomes other than $14 \mathrm{q}$, such as chromosome $1,^{26} 18^{27}$ and $22 .^{27,28}$ To the best of our knowledge, this is the first report in which high-resolution a-CGH is applied to the analysis of chromosome 14 in meningiomas. The increased resolution of a-CGH is directly related to the development of thousands of BAC clones that allow for the analysis of the entire human genome including chromosome $14^{23}$ and the availability of overlapping BAC clones for each chromosome region of interest. Adjacent and overlapping clones can be used together to increase the efficiency and accuracy of the analysis, as the tile-path array in which a set of overlapping clones are used, deletions or gains larger than or equal to a single clone, frequently involve also neighbouring clones. ${ }^{21}$ In this study, we used a total of 807 BAC clones to map the long arm of chromosome 14; with such clones, insert sizes of between 100 and $300 \mathrm{~kb}$ were obtained which would allow the identification of altera- 

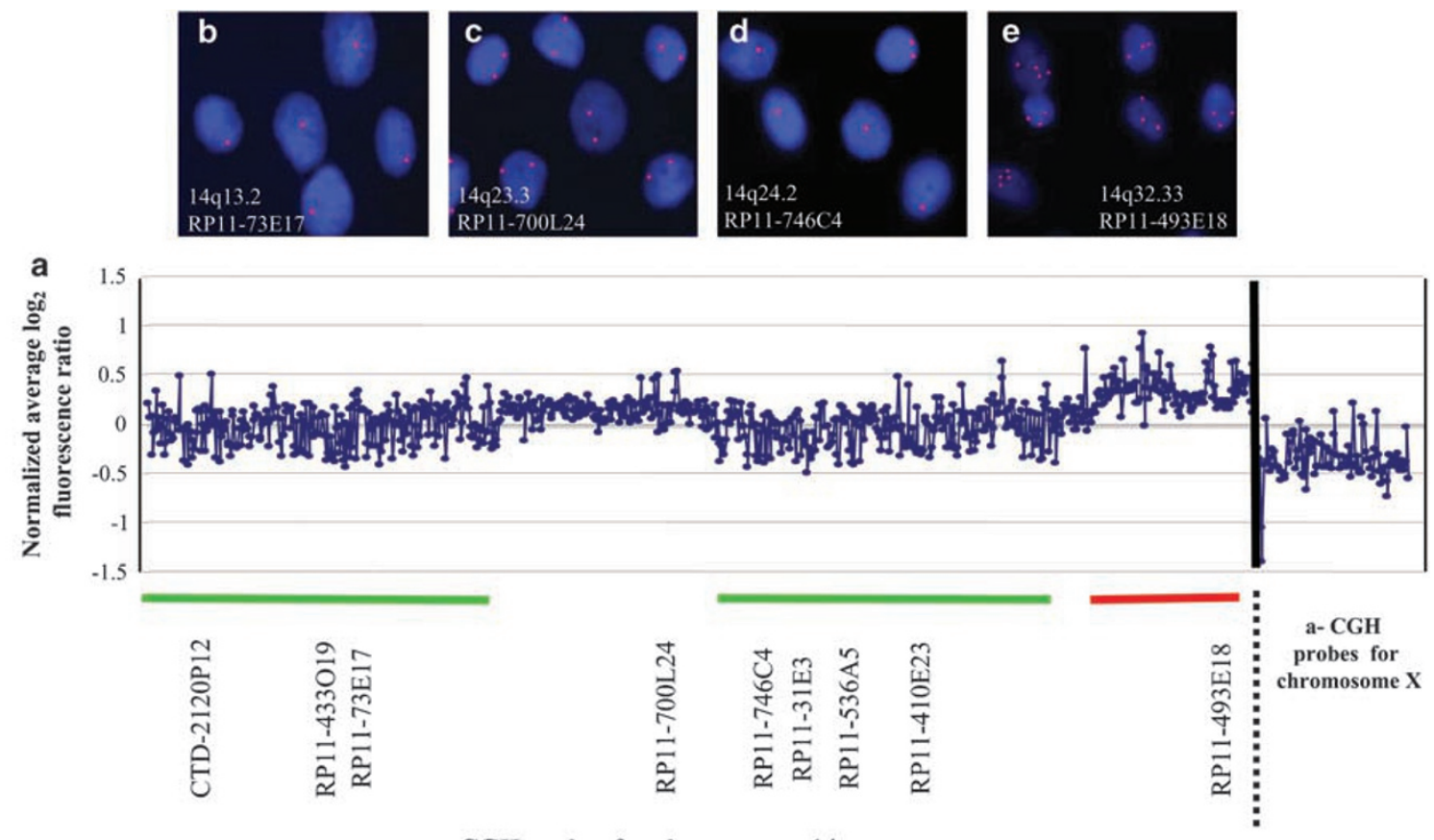

a- CGH probes for chromosome 14q

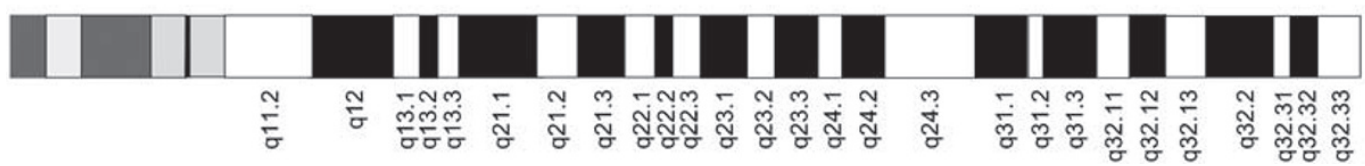

Figure 3 Ideogram of chromosome 14q from a meningioma tumour (case 9) where green and red lines highlight the deleted and gained chromosome regions as detected by a-CGH, respectively (a) and confirmatory iFISH results (b-e). As illustrated, a-CGH from this tumour showed losses at 14q11.2-q21.2 and 14q24.1-q32.12 (a); a single iFISH signal consistent with chromosome 14 deletion was also detected for both chromosome 14q regions with the RP11-73E17 (14q13.2) and RP11-746C4 (14q24.2) BAC clone FISH probes (b and d, respectively). In turn, the RP11-700L24 (14q23.3) BAC clone (c) displayed a normal diploid iFISH pattern, while the RP11-493E18 BAC clone (e) was associated with three and four hybridization signals, indicating the existence of an increased number of copies in the 14q32.33 region, in line with what was observed by a-CGH (a). Other smaller losses were interpreted as being potentially associated with copy number variation.

tions in relatively small regions. To avoid overinterpretation of changes at single clones due to the technical artefacts, ${ }^{18} \mathrm{CNV}$ were only defined when altered fluorescence ratios were observed in two or more consecutive clones. The use of both constitutional and normal female meningeal cells' DNA, as reference DNA in the hybridization, in addition to a DNA pool of PB cells from healthy individuals, further contributed to decrease the potential occurrence of either false-positive or false-negative results due to the differences in tumour and reference DNA.

Overall, a high degree of agreement was observed for the meningioma tumours analysed between a-CGH and iFISH results, with a commercially available probe identifying the IGH gene locus at chromosome 14q32.3. However, it should be noted that the use of a-CGH and confirmatory iFISH assays with some of the a-CGH probes used allowed a more detailed definition of the abnormalities identified, additional alterations being detected in a few meningiomas. Of note, chromosome $14 \mathrm{q}$ abnormalities could be confirmed by a-CGH even in cases where these alterations were present in as low as between $20-30 \%$ of all cells in the sample, as counted by iFISH. Recently, a similar chromosome 14 tiling-path a-CGH has been applied to the study of a series of gastrointestinal stromal tumours (GISTs) with a comparable sensitivity, allowing for the unequivocal detection of genomic changes present in samples with $\geqslant 40 \%$ tumour cells. $^{29}$ Overall, high-resolution a-CGH confirmed that the most characteristic alteration of chromosome 14 in meningioma tumour is the entire loss of this chromosome. Interestingly, in addition to those cases showing monosomy 14 by iFISH, an a-CGH profile consistent with monosomy 14 was also detected in a patient showing two FISH signals per nuclei with both the 14q32.3 probe and the BAC clones tested. Such apparent discrepancy could be due to the occurrence of tetraploidization of an already undetectable tumour cell clone carrying monosomy 14 , as in this tumour, a tetraploid profile was observed by iFISH for all chromosomes other than chromosome 14 (data not shown), suggesting the hemizygous nature of this chromosome. 
Discrepant a-CGH vs iFISH profiles were detected in another two cases. In one patient, an apparently diploid DNA content was detected for all chromosome $14 \mathrm{q}$ BAC clones by a-CGH; however, iFISH analyses of the tumour cells with the same BAC probes confirmed the presence of four copies of chromosome $14 \mathrm{q}$ in the context of a tetraploid karyotype. At present, it is well known that polyploidy affects the assessment of DNA copy number by a-CGH; ${ }^{18,21}$ because of this, in such cases, a-CGH results should be corrected based on the results found with other techniques such as karyotyping and FISH. ${ }^{21}$ In the second discrepant case, coexisting gains and losses of different chromosome $14 \mathrm{q}$ regions were detected by a-CGH. Coexistence of regional losses and gains of genetic material of chromosome $14 \mathrm{q}$ may reflect the occurrence of complex structural abnormalities involving different regions of this chromosome. This hypothesis would be supported by the length of the aberrations encountered in this tumour, which affected hundreds of clones containing a relatively high number of genes. In line with this, previous studies have shown the occurrence of structural abnormalities of chromosome 14 in a small proportion of all meningiomas $^{25,30-35}$ such alterations consisting of translocations involving chromosome 14 and several other different chromosomes, particularly chromosomes 1 and 22 25,32-35 Unfortunately, in this study, we could not confirm the exact underlying chromosome changes present in this patient (nuc ish (DXZ1x1), (DYZ3x1), (D1Z2x1), (D1Z5x3), (D7Z1x2), (ABLx2), (D10Z1x2), (CCND1x2), (IGHx3), (PMLx3), (RARAx2), (BCL2x4) and (BCRx1) [200]).

From the clinical point of view, although a few number of recurrences had been observed at the end of the study, they were restricted to patients carrying the complete loss of chromosome 14 . Recently, we have shown that meningioma patients with monosomy 14 and del(1p36), together with a larger tumour size, have a higher probability of relapsing during the first 2.5 years after diagnostic surgery. ${ }^{10}$ Interestingly, in our study, all except two cases with the complete loss of chromosome 14 also displayed del(1p36) in the ancestral tumour cell clone (Table 1) and these latter patients included the two cases who had relapsed. Previous studies ${ }^{1,11,36}$ have defined a few regions (eg, 14q24.3-q32.33 and 14q11.2) as critical regions in chromosome 14 where candidate genes involved in determining the clinical behaviour of meningiomas could be localized. In this study, we were not able to identify small interstitial deletions, mutations or epigenetic silencing phenomena involving these regions, in the series of patients analysed. However, further studies on larger tumour series might allow the identification of some few cases displaying partial deletions on chromosome 14, which would provide valuable information on the critical regions of this chromosome harbouring genes involved in the development and progression of meningiomas.
In summary, in this study, we report for the first time the high-resolution a-CGH profiles of chromosome $14 \mathrm{q}$ in meningiomas. Our results confirm that the complete loss of chromosome $14 \mathrm{q}$ is the most frequent alteration associated with this chromosome, while other abnormalities consisting of either gains of the entire chromosome or coexistence of complex gains and losses of different regions of chromosome $14 \mathrm{q}$ could be only sporadically detected.

\section{Acknowledgements}

This work has been partially supported by grants from Consejería de Sanidad (44-05) Junta de Castilla y León (Valladolid, Spain; Fondo de Investigaciones Sanitarias (FIS/FEDER 06/0312 and RTICC RD06/ 0020/0035 from the Instituto de Salud Carlos III, Ministerio de Sanidad y Consumo, Madrid, Spain) and Fundación MMA (Madrid, Spain). MD Tabernero is supported by IECSCYL. AB Espinosa is supported by grant FI05/00266 from the Instituto de Salud Carlos III (Ministerio de Sanidad y Consumo, Madrid, Spain). Normal meningeal samples were provided by the Banco de Tejidos Neurológicos de Navarra (Pamplona, Spain) by C Caballero and T Tuñón. Normal DNA pool from female and male volunteers were provided by National DNA Bank of Spain, Salamanca, Spain (www.bancoadn.org).

\section{References}

1 Simon M, von Deimling A, Larson JJ et al: Allelic losses on chromosomes 14,10 , and 1 in atypical and malignant meningiomas: a genetic model of meningioma progression. Cancer Res 1995; 55: 4696-4701.

2 Weber RG, Bostrom J, Wolter $M$ et al: Analysis of genomic alterations in benign, atypical, and anaplastic meningiomas: toward a genetic model of meningioma progression. Proc Natl Acad Sci USA 1997; 94: 14719-14724.

3 Cai DX, Banerjee R, Scheithauer BW, Lohse CM, KleinschmidtDemasters BK, Perry A: Chromosome 1p and 14q FISH analysis in clinicopathologic subsets of meningioma: diagnostic and prognostic implications. J Neuropathol Exp Neurol 2001; 60: 628-636.

4 Al-Mefty O, Kadri PA, Pravdenkova S, Sawyer JR, Stangeby C, Husain M: Malignant progression in meningioma: documentation of a series and analysis of cytogenetic findings. J Neurosurg 2004; 101: 210-218.

5 Tabernero MD, Espinosa AB, Maillo A et al: Characterization of chromosome 14 abnormalities by interphase in situ hybridization and comparative genomic hybridization in 124 meningiomas: correlation with clinical, histopathologic, and prognostic features. Am J Clin Pathol 2005; 123: 744-751.

6 Tabernero MD, Espinosa AB, Maillo A et al: Patient gender is associated with distinct patterns of chromosomal abnormalities and sex chromosome linked gene-expression profiles in meningiomas. Oncologist 2007; 12: 1225-1236.

7 Menon AG, Rutter JL, von Sattel JP et al: Frequent loss of chromosome 14 in atypical and malignant meningioma: identification of a putative 'tumor progression' locus. Oncogene 1997; 14: $611-616$.

8 Lamszus K, Kluwe L, Matschke J, Meissner H, Laas R, Westphal M: Allelic losses at $1 \mathrm{p}, 9 \mathrm{q}, 10 \mathrm{q}, 14 \mathrm{q}$, and $22 \mathrm{q}$ in the progression of aggressive meningiomas and undifferentiated meningeal sarcomas. Cancer Genet Cytogenet 1999; 110: 103-110.

9 Maillo A, Orfao A, Sayagues JM et al: New classification scheme for the prognostic stratification of meningioma on the basis of chromosome 14 abnormalities, patient age, and tumor histopathology. J Clin Oncol 2003; 21: 3285-3295.

10 Maillo A, Orfao A, Espinosa $\mathrm{AB}$ et al: Early recurrences in histologically benign/grade I meningiomas are associated with 
large tumors and coexistence of monosomy 14 and $\operatorname{del}(1 \mathrm{p} 36)$ in the ancestral tumor cell clone. Neuro Oncol 2007; 9: 438-446.

11 Lusis EA, Watson MA, Chicoine MR et al: Integrative genomic analysis identifies NDRG2 as a candidate tumor suppressor gene frequently inactivated in clinically aggressive meningioma. Cancer Res 2005; 65: 7121-7126.

12 Pinkel D, Segraves R, Sudar D et al: High resolution analysis of DNA copy number variation using comparative genomic hybridization to microarrays. Nat Genet 1998; 20: 207-211.

13 Kallioniemi A, Kallioniemi OP, Sudar D et al: Comparative genomic hybridization for molecular cytogenetic analysis of solid tumors. Science 1992; 258: 818-821.

14 Arslantas A, Artan S, Oner U et al: Comparative genomic hybridization analysis of genomic alterations in benign, atypical and anaplastic meningiomas. Acta Neurol Belg 2002; 102: 53-62.

15 Pelz AF, Klawunde P, Skalej $\mathrm{M}$ et al: Novel chromosomal aberrations in a recurrent malignant meningioma. Cancer Genet Cytogenet 2007; 174: 48-53.

16 Zang KD: Meningioma: a cytogenetic model of a complex benign human tumor, including data on 394 karyotyped cases. Cytogenet Cell Genet 2001; 93: 207-220.

17 van Tilborg AA, Al Allak B, Velthuizen SC et al: Chromosomal instability in meningiomas. J Neuropathol Exp Neurol 2005; 64: $312-322$

18 Snijders AM, Nowak N, Segraves R et al: Assembly of microarrays for genome-wide measurement of DNA copy number. Nat Genet 2001; 29: 263-264.

19 Cai WW, Mao JH, Chow CW, Damani S, Balmain A, Bradley A: Genome-wide detection of chromosomal imbalances in tumors using BAC microarrays. Nat Biotechnol 2002; 20: 393-396.

20 Fiegler H, Carr P, Douglas EJ et al: DNA microarrays for comparative genomic hybridization based on DOP-PCR amplification of BAC and PAC clones. Genes Chromosomes Cancer 2003; 36: $361-374$.

21 Vermeesch JR, Melotte C, Froyen G et al: Molecular karyotyping: array CGH quality criteria for constitutional genetic diagnosis. J Histochem Cytochem 2005; 53: 413-422.

22 Jones DT, Ichimura K, Liu L, Pearson DM, Plant K, Collins VP: Genomic analysis of pilocytic astrocytomas at $0.97 \mathrm{Mb}$ resolution shows an increasing tendency toward chromosomal copy number change with age. J Neuropathol Exp Neurol 2006; 65: 1049-1058.

23 Ishkanian AS, Malloff CA, Watson SK et al: A tiling resolution DNA microarray with complete coverage of the human genome. Nat Genet 2004; 36: 299-303.
24 Louis DN, Ohgaki H, Wiestler OD et al: The 2007 WHO classification of tumours of the central nervous system. Acta Neuropathol 2007; 114: 97-109.

25 Lopez-Gines C, Cerda-Nicolas M, Gil-Benso R et al: Association of loss of $1 \mathrm{p}$ and alterations of chromosome 14 in meningioma progression. Cancer Genet Cytogenet 2004; 148: 123-128.

26 Buckley PG, Jarbo C, Menzel U et al: Comprehensive DNA copy number profiling of meningioma using a chromosome 1 tiling path microarray identifies novel candidate tumor suppressor loci. Cancer Res 2005; 65: 2653-2661.

27 Nunes F, Shen Y, Niida Y et al: Inactivation patterns of NF2 and DAL-1/4.1B (EPB41L3) in sporadic meningioma. Cancer Genet Cytogenet 2005; 162: 135-139.

28 Hansson CM, Buckley PG, Grigelioniene G et al: Comprehensive genetic and epigenetic analysis of sporadic meningioma for macro-mutations on $22 \mathrm{q}$ and micro-mutations within the NF2 locus. BMC Genomics 2007; 8: 16.

29 Wozniak A, Sciot R, Guillou L et al: Array CGH analysis in primary gastrointestinal stromal tumors: cytogenetic profile correlates with anatomic site and tumor aggressiveness, irrespective of mutational status. Genes Chromosomes Cancer 2007; 46: 261-276.

30 Albrecht S, Goodman JC, Rajagopolan S, Levy M, Cech DA, Cooley LD: Malignant meningioma in Gorlin's syndrome: cytogenetic and p53 gene analysis. Case report. I Neurosurg 1994; 81: 466-471.

31 Henn W, Cremerius U, Heide G et al: Monosomy $1 \mathrm{p}$ is correlated with enhanced in vivo glucose metabolism in meningiomas. Cancer Genet Cytogenet 1995; 79: 144-148.

32 Zattara-Cannoni H, Gambarelli D, Dufour H et al: Contribution of cytogenetics and FISH in the diagnosis of meningiomas. A study of 189 tumors. Ann Genet 1998; 41: 164-175.

33 Sawyer JR, Husain M, Pravdenkova S, Krisht A, Al-Mefty O: A role for telomeric and centromeric instability in the progression of chromosome aberrations in meningioma patients. Cancer 2000; 88: $440-453$

34 Cerda-Nicolas M, Lopez-Gines C, Perez-Bacete M, Barcia-Salorio JL, Llombart-Bosch A: Histopathological and cytogenetic findings in benign, atypical and anaplastic human meningiomas: a study of 60 tumors. Clin Neuropathol 2000; 19: 259-267.

35 Prowald A, Wemmert S, Biehl C et al: Interstitial loss and gain of sequences on chromosome 22 in meningiomas with normal karyotype. Int J Oncol 2005; 26: 385-393.

36 Leone PE, Bello MJ, de Campos JM et al: NF2 gene mutations and allelic status of $1 \mathrm{p}, 14 \mathrm{q}$ and $22 \mathrm{q}$ in sporadic meningiomas. Oncogene 1999; 18: 2231-2239. 